\title{
Simulation of $Z$ plus graviton/unparticle production at the LHC
}

\author{
Stefan Ask ${ }^{\mathrm{a}}$ \\ School of Physics \& Astronomy, University of Manchester, Manchester M13 9PL, UK
}

Received: 13 January 2009 / Revised: 5 February 2009 / Published online: 25 February 2009

(C) Springer-Verlag / Società Italiana di Fisica 2009

\begin{abstract}
Theories with extra dimensions have gained much interest in recent years as candidates for a possible extension of the SM. The observation of large extra dimensions through real graviton emission is one of the most popular related new phenomena. The main experimental signatures from graviton emission are production of single jet and single photon events, which have been studied in great detail. This work describes the implementation of graviton production together with either a $Z$ or a photon in Pythia 8 . The potential of using $Z$ plus graviton production at the LHC as a complementary channel is also studied. For completeness, this work also includes the more recently proposed scenario of unparticle emission, since the effective theory of unparticles to some extent represents a generalization of the large extra dimension model.
\end{abstract}

\section{Introduction}

The possibility of observing gravity in extra dimensions (ED) at the $\mathrm{TeV}$ scale was proposed a few years ago and has since gained a large interest. One of the first proposals was the so-called ADD scenario [1,2] which suggests that gravity alone would propagate in large extra dimensions (LED). This would explain the weakness of gravity experienced by the SM fields, since they only would have access to the normal 4 dimensional space-time. This scenario has been studied in detail [3, 4] and dedicated searches have been performed at different experiments, [5-12]. The large extra dimensions in the ADD scenario have a flat space topology and, for this reason, astrophysical observations imply stringent constraints in the case of a small number of extra dimensions. It has, however, later been shown that these constraints would be evaded if space-time is slightly warped, as in the popular RS models [13]. In this case the graviton $(G)$ would acquire a small effective mass, which makes the model [14] insensitive to astrophysical constraints from

a e-mail: Stefan.Ask@manchester.ac.uk low-energy processes. ${ }^{1}$ Then even one large extra dimension could have escaped the present experimental measurements [15].

Recently a so-called unparticle $(U)$ model has also gained much attention $[16,17]$. This relates to phenomena from a scale invariant sector which is coupled to the SM by a connector sector with a high mass scale. This scenario is normally considered to be less well motivated than extra dimensional gravity, which could solve the so-called hierarchy problem and is also motivated by string theory. It could, however, imply unusual experimental signatures at the LHC which should not be missed. In addition, the unparticle model is, from a phenomenological point of view, a generalization of the large extra dimension case and therefore both cases can to some extent be covered at the same time.

The main experimental signatures from both gravity in large extra dimensions as well as the unparticle model would be an excess of single jet and single photon events. In this work we describe the implementation of $Z / \gamma+G / U$ production as a so-called semi-internal process to Pythia 8 $[18,19]$. Furthermore $Z+G / U$ production at the LHC is studied, since this process could provide a complement in order to constrain the model if a signal would be observed in the main channels.

The next section gives a reminder of the relevant model parameters and, in order to simplify comparison between different papers, the most common conventions are summarized. This is followed by the implementation of the process and the validation tests that have been performed. Finally a study at generator level of the $p p \rightarrow Z+G / U$ cross section predicted at the LHC is presented, including a relative comparison with the similar SM process $p p \rightarrow Z Z \rightarrow \ell \ell v v$.

\section{The models and parameters}

The extra dimension model considered in this work is the same as that described in [3]. Here all SM fields are con-

\footnotetext{
${ }^{1}$ The high-energy phenomena relevant at collider experiments are, however, unchanged.
} 
fined to a 4 dimensional brane present in a larger dimensional space where only gravity can propagate. It is assumed that the brane is rigid so that effects from fields related to the brane dynamics can be neglected. The extra dimensions would be compactified with a radius $R$. In the case of real graviton production at the LHC the momentum component of the graviton in the extra dimensions would be observed on the brane as a mass. The finite size of the ED implies a discrete series of allowed mass modes, i.e. the so-called Kaluza-Klein (KK) tower. The graviton is coupled to the SM fields through the energy-momentum tensor as,

$\Delta \mathcal{L}=-\left(8 \pi G_{N}\right)^{\frac{1}{2}} G_{\mu \nu}^{(i)} T^{\mu \nu}$

where $G_{N}$ is the Newton constant and $i$ is the KK mode index. For the graviton production cross section the extremely weak coupling to gravity is compensated by the additional phase space in the extra dimensions. For this reason gravitational phenomena would appear at energies around the $D$ dimensional fundamental scale of gravity, $M_{D}$. The size of the ED in this scenario is allowed by experimental data to be almost as large as a millimeter [6]. Since the KK mode separation relates to the size as $\Delta m \sim 1 / R$, the discrete $\mathrm{KK}$ series can be approximated by a continuous spectrum. In order to simplify the calculations, the extra dimensions are assumed to have the geometry of a $n$-dimensional torus. In this model of gravity in large extra dimensions, the size of the extra dimensions $(R)$ and the fundamental scale of gravity $\left(M_{D}\right)$ are related to the Newton constant for $n$ extra dimensions as,

$G_{N}^{-1}=C \cdot R^{n} M_{D}^{n+2}$

where the exact definitions vary slightly between different papers. This work follows the parameter definitions used in [3] where $C=8 \pi$. However, to simplify for comparison between papers the different conventions are summarized. In the papers $[3,4,20]$ the fundamental scale of gravity is referred to as $M_{D}, M$ and $M_{S}$. These are the three most common definitions and are related as follows,

$M^{n+2}=2 M_{D}^{n+2}$

$M_{S}^{n+2}=8 \pi^{1-\frac{n}{2}} \Gamma\left(\frac{n}{2}\right) M_{D}^{n+2}$

The work in [20] also defines the size $R$ slightly different compared to [3, 4], $R_{S}=2 \pi R_{D}$. From these relations the different expressions in the literature for the integral over the very dense KK states reduce to,

$\sum_{k} \rightarrow \int \mathrm{d} m^{2} \frac{\pi^{n / 2} \cdot R_{D}^{n} \cdot\left(m^{2}\right)^{n / 2-1}}{\Gamma\left(\frac{n}{2}\right)}$

Here $k$ is the graviton momentum component in the extra dimensions and $m^{2}$ is the graviton mass squared.
The unparticle model studied here is described in [17]. In this scenario the so-called unparticles originate from a scale invariant sector with a non-trivial fixed point. This sector interacts with the SM fields through a connector sector with a high mass scale, $M_{U}$. Renormalization effects in the scale invariant sector give rise to dimensional transmutation of the unparticles at an energy scale $\Lambda_{U}$. This transmutation is determined by a scale dimension parameter $d_{U}$ which has to be greater than one due to unitarity arguments, but is allowed to take non-integer values. This scenario gives rise to quite unusual phenomena. In terms of real unparticle emission it implies that the unparticle would not have a fixed invariant mass squared, but instead an invariant mass spectrum. The unparticle would also appear with a $d_{U}$-body final state phase space, which would give rise to an unusual missing energy signature. In accordance with [17] only the production of unparticles from the allowed spin- 1 and spin- 2 effective unparticle operators is considered here and the unparticles are assumed to be SM singlets. In this model the cross sections are determined by the three unparticle parameters, $d_{U}, \Lambda_{U}$ and $\lambda$. Here $\lambda$ is an effective coupling between the unparticle and SM operators which is related to the connector scale $M_{U}$. In the case of spin-2 unparticles two different effective operators are allowed which are associated with the independent coupling constants $\lambda$ and $\lambda^{\prime}$.

As pointed out in [17] the effective theory of spin-2 unparticle emission with $\lambda=\lambda^{\prime}$ is, apart from constant factors, equivalent to graviton emission in large extra dimensions. Here the invariant mass spectrum from the dimensional transmutation of the unparticles is identical to the dense KK tower of the large extra dimensions. For this reason the graviton emission process can be seen as a special case of the unparticle model and, as will be described below, both scenarios can be covered by the same implementation with only minor changes. This has the advantage of allowing the more motivated extra dimension search to be extended to cover the unparticle case. To cover both scenarios at once could be of great value in the case where future developments discover new and perhaps even more interesting analogies between the unparticle picture and gravity in extra dimensions. A potential connection between a noninteger $d_{U}$ value and so-called warped extra dimensions has for example been addressed in [21].

\section{Process implementation}

The process $f \bar{f} \rightarrow Z+G / U$ was implemented ${ }^{2}$ as a semiinternal process to Pythia 8.108. It contains a 2-to-2 parton

\footnotetext{
${ }^{2}$ As a by-product also the process $f \bar{f} \rightarrow \gamma+G / U$ was implemented in a separate class. This corresponds to the photon limit of the $Z$ process as described later in the text.
} 
level process class which can be used by the main Pythia library. The class is implemented with the same structure as used for the internal parton level processes and is, therefore, also used in the same way as the internal classes inside the Pythia library. No $Z / \gamma^{*}$ interference effects were taken into account and the $Z$ decays isotropically. The location and details of the software can be found in the Appendix.

The differential cross section used corresponds to

$$
\begin{aligned}
& \frac{\mathrm{d} \sigma}{\mathrm{d} p_{U}^{2} \mathrm{~d} t}(f \bar{f} \rightarrow Z+U) \\
& \quad=\frac{|\overline{\mathcal{M}}|^{2}}{16 \pi \cdot s^{2}} \cdot \frac{A\left(d_{U}\right)}{2 \pi \cdot \Lambda_{U}^{2}}\left(\frac{p_{U}^{2}}{\Lambda_{U}^{2}}\right)^{d_{U}-2} \theta\left(p_{U}^{0}\right) \theta\left(p_{U}^{2}\right)
\end{aligned}
$$

where $p_{U}^{2}$ is the invariant mass squared. Apart from the matrix element, the first term only contains standard 2-to2 scattering phase space factors. The remaining terms contain the phase space and final state mass spectrum of the unparticle. Here, $A\left(d_{U}\right)$ is a normalization constant of the $d_{U}$-body phase space. The full spin- and color-averaged matrix elements given by (42) and (47) in [17] are used. This corresponds to the most general form of the matrix element from the two allowed effective spin- 2 unparticle operators. The spin-1 case only includes the contribution from the vectorial operator. This is sufficient when considering unpolarized particle beams, since including the second axialvectorial operator would result in the same formula but with $\lambda \rightarrow \sqrt{\lambda_{v}^{2}+\lambda_{a}^{2}}$.

The variable mass spectrum shown in equation (3.2) gave rise to the main difference with respect to the internal processes available in Pythia. It turned out, however, to be conveniently implemented by re-weighting an internally produced Breit-Wigner distribution. By using the graviton already available in Pythia, the unparticle masses are generated according to a Breit-Wigner which is re-weighted to a flat distribution. The invariant mass measure of the cross section, $\left(p_{U}^{2}\right)^{d_{U}-2} d p_{U}^{2}$, then ensures that the final events are generated with the correct mass distribution. In order to achieve a high MC efficiency one has to take care to match the bulk of the Breit-Wigner to the bulk of the unparticle distribution. It should be pointed out that this only affects the speed of generating events, by throwing events more often in the region where the differential cross section is large, and not the final cross section or event properties.

The same formula and code is used for producing graviton events. In this case $\lambda=\lambda^{\prime}=1$ is used independent of the input values and the $d_{U}$-body phase space factor is changed to,

$$
\begin{gathered}
A\left(d_{U}\right)=\frac{16 \pi^{2} \sqrt{\pi}}{(2 \pi)^{2 d_{U}}} \frac{\Gamma\left(d_{U}+\frac{1}{2}\right)}{\Gamma\left(d_{U}-1\right) \Gamma\left(2 d_{U}\right)} \\
\rightarrow \quad S^{\prime}(n)=\frac{2 \pi \cdot \pi^{n / 2}}{\Gamma\left(\frac{n}{2}\right)}
\end{gathered}
$$

The remaining input arguments are used in the following way,

$d_{U}=\frac{n}{2}+1$

$\Lambda_{U}=M_{D}$

which implies that in total only two constants have to be changed in order to switch between the models.

A truncation option was also implemented in order to test the validity of the effective theory. In order to trust the perturbative calculations of the effective theory the $\hat{s}$ of the process should be smaller than the fundamental mass scale of the theory, $\Lambda_{U}$ or $M_{D}$. This does not have to be the case at the LHC where the collision energy is $14 \mathrm{TeV}$ and, for example, the $M_{D}$ scale at present does not have to be larger than about $1 \mathrm{TeV}$. For this reason the truncation functionality suppresses the cross section at $\hat{s}>\Lambda_{U}^{2}$ by a factor $\Lambda_{U}^{4} / \hat{s}^{2}$. This truncation also implies that the mass spectrum is suppressed at large values. The truncation effect becomes increasingly significant with large $d_{U}$ values, since the mass spectrum then becomes peaked towards higher values. This is illustrated in Fig. 3.1, which shows the graviton mass
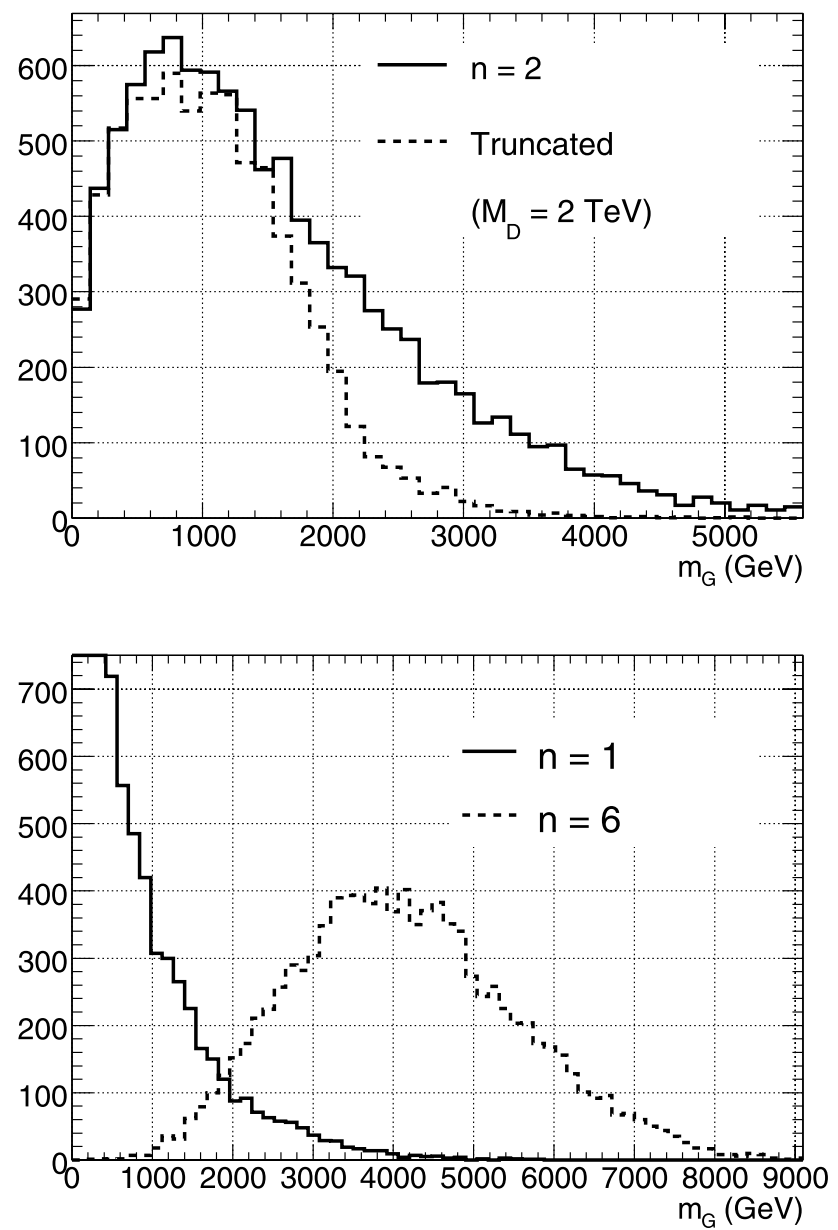

Fig. 3.1 The graviton mass spectrum for $n=2$ with and without truncation (upper plot) as well as for $n=1$ and 6 with no truncation (lower plot) 
spectrum for $n=2$ with and without truncation (upper plot) as well as for $n=1$ and 6 with no truncation (lower plot). The truncation in the case $n=2$ is using $M_{D}=2 \mathrm{TeV}$. In a similar way the truncation effect becomes increasingly important with an increased transverse energy requirement of the event selection in the analysis.

The truncation option was primarily used to verify that the difference between the cross section obtained with and without truncation is negligible. This implies that the uncontrolled $\hat{s}$-region does not contribute significantly. However, in the case of a discrepancy, the truncated cross section can still be used as a conservative estimate from the region which is under perturbative control.

\section{Validation cross checks}

In order to validate the different parts of the implementation, the results were checked against several similar processes:

- $e^{+} e^{-} \rightarrow \gamma+G$ in $[3,4]$

- $p p \rightarrow \gamma+G$ in [3];

- $e^{+} e^{-} \rightarrow Z+G$ in $[14,20]$

- $e^{+} e^{-} \rightarrow \gamma+U$ in [17].

Together these should provide sufficient cross checks to validate all parts of the implemented process. Most parts are covered by reproducing the results in $[3,4]$ from the first two processes. The third processes verifies the $Z$ specific parts of the spin- 2 matrix element. The fourth process verifies that the unparticle phase space factors and spin- 1 matrix element are correct. The only part which is not directly tested is the matrix element in the case of a spin-2 unparticle with $\lambda \neq \lambda^{\prime}$. On the other hand, when setting $\lambda=\lambda^{\prime}$ a specific cancellation is required in order to reproduce the $Z+G$ matrix element and therefore this is considered to be a good general test.

The same code was also used to produce the photon results. The photon limit of the $Z+G / U$ process was obtained by making the following changes,

$m_{Z} \rightarrow 0$

$\frac{g_{v}^{2}+g_{a}^{2}}{4} \rightarrow Q^{2}$

$\frac{g}{\cos \theta_{W}} \rightarrow e$

where the following coupling conventions were used, $g_{v}=$ $-\frac{1}{2}+2 \sin ^{2} \theta_{W}$ and $g_{a}=-\frac{1}{2}$. The fact that the photon limit of the $Z$ matrix element is checked is also a valuable cross check of the implementation.

\section{$5 Z+G / U$ production at the $\mathrm{LHC}$}

The potential of using $Z+G / U$ events to confirm a signal observed in the main channels was also studied. These events would give rise to di-leptons and large missing transverse energy. The most favorable event topology from an experimental point of view is when the leptons are either an electron or muon pair. The SM process with the most similar experimental signature is $Z Z$ production, where one $Z$ decays into electrons or muons and the other into neutrinos, $Z Z \rightarrow \ell \ell v v$. For this reason it is interesting to investigate the possible $Z+G / U$ cross section relative to this $\mathrm{SM} Z Z$ process.

The ATLAS $Z Z \rightarrow \ell \ell v v$ analysis presented in [22] will be used as a reference through this study. This analysis predicts an overall inclusive $Z Z$ cross section of $\sigma_{Z Z}=$ $14.8 \mathrm{pb}$, which was obtained by the MC@NLO generator and using the CTEQ 6M parton distribution functions (PDFs). The corresponding cross section obtained by Pythia 8 was $11.4 \mathrm{pb}$ using the CTEQ5L PDFs. This appears consistent for a comparison between leading order (LO) and next-toleading order (NLO) results.

The $Z Z$ events generated by Pythia were then passed through a similar event selection to the one used in [22]. This corresponds to the following cuts:

1) Opposite charged electrons or muons;

2) $p_{T}^{\ell}>20 \mathrm{GeV}$

3) $\left|\eta^{\ell}\right|<2.5$

4) $\left|M_{\ell \ell}-91.2\right|<10 \mathrm{GeV}$;

5) $p_{T}(Z)>100 \mathrm{GeV}$.

The first three requirements ensure that there are two high$p_{T}$ electrons or muons inside the central part of the experiment. The remaining two cuts imply that the leptons are consistent with the decay of an on-shell $Z$ with high $p_{T}$. After applying this selection to the generator level $Z Z$ events from Pythia, a selected cross section of $14 \mathrm{fb}$ was obtained. This value also appears reasonable compared to the $10.2 \pm 0.2 \mathrm{fb}$ obtained in the full ATLAS analysis, when considering that they are calculated at LO and NLO respectively, and that the ATLAS analysis includes the full ATLAS detector simulation and reconstruction chain.

Since the existing limits on $M_{D}$ exclude values between approximately 0.5 and $1.5 \mathrm{TeV}$ the following parameter values were used as benchmark points for the $Z+G$ study:

- $n=1,2,3,6$;

- $M_{D}=2,2.5,3 \mathrm{TeV}$.

Events generated with, $n=2, M_{D}=2 \mathrm{TeV}$ and using the truncation option were passed through the $Z Z$ selection described above and a selected cross section of $2.6 \mathrm{fb}$ was obtained. This shows that for this particular point the $Z+G$ signal would amount to about $20 \%$ of the expected SM $Z Z \rightarrow \ell \ell v v$ signal.

Figure 5.1 shows the selected cross section for the different benchmark points and the results are shown with and without truncation. One can clearly see that the cross section increases with a larger number of extra dimensions, but 

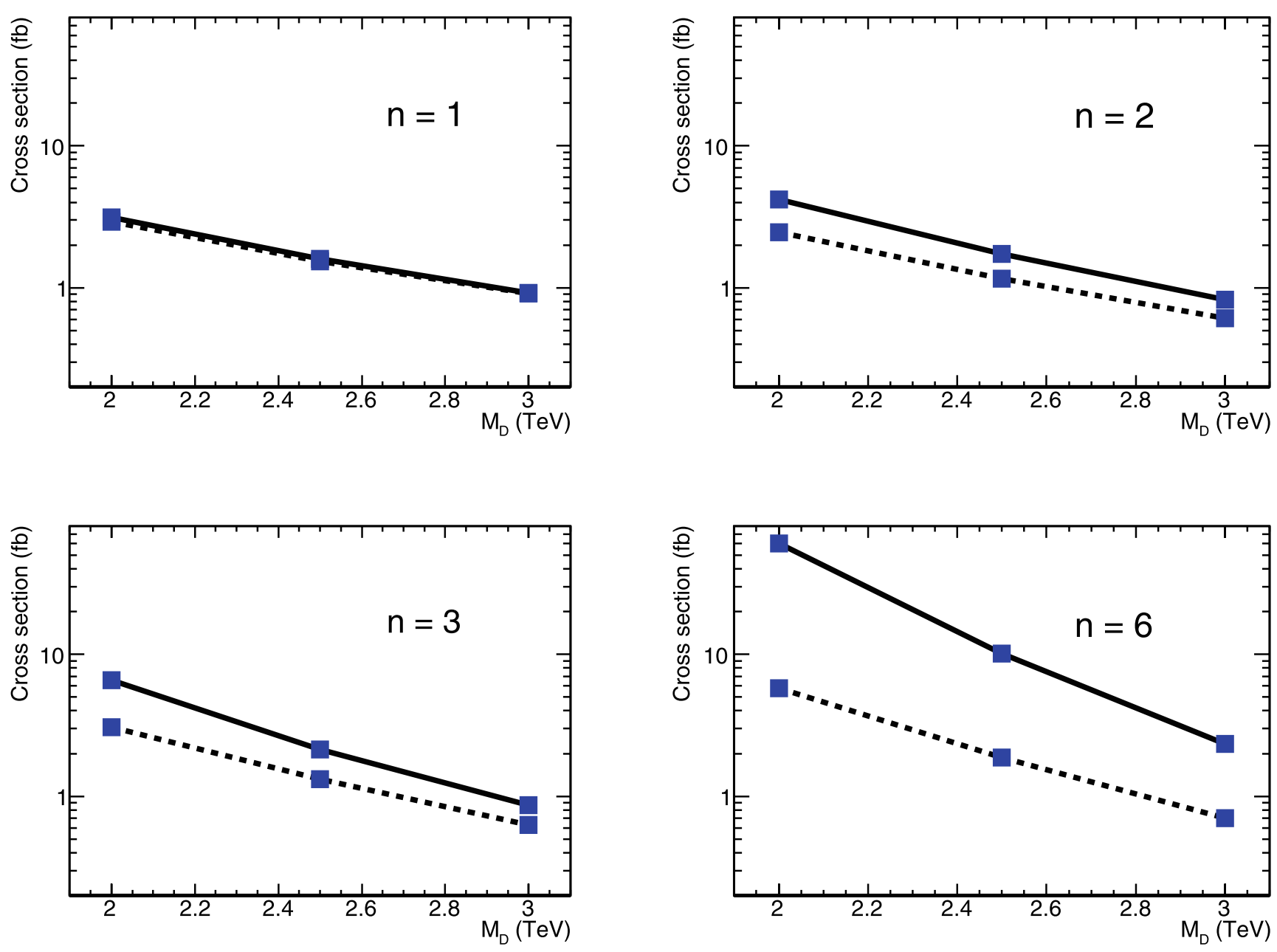

Fig. 5.1 Selected $Z+G$ cross section as a function of $M_{D}$. The results are shown with (dashed line) and without (solid line) truncation

at the same time an increasing fraction moves into the nonperturbative $\hat{s}$ region. For this reason the effective theory is not trustworthy at large $n$. At small $n$, on the other hand, the effective theory is fairly valid and the $Z+G$ channel could be used to cross check a signal observed in the more conventional channels. This will, however, require relatively large amounts of data. Assuming for example $\sigma_{b}=20 \mathrm{fb}$ of selected background ${ }^{3}$ in total and a signal of $\sigma_{s}=2.6 \mathrm{fb}$, a data sample in the order of,

$\mathcal{L}=\frac{25 \cdot \sigma_{b}}{\sigma_{s}^{2}} \sim 75 \mathrm{fb}^{-1}$

would be necessary for a $5 \sigma$ discovery.

The event selection could be improved to increase the sensitivity of the graviton signal. The upper plot of Fig. 5.2 shows the $p_{T}(Z)$ distributions of the SM $Z Z \rightarrow \ell \ell v v$ process and the $Z+G$ process with $n=2$ and $M_{D}=2 \mathrm{TeV}$.

${ }^{3}$ This background value is assumed since the ATLAS study suggests about $50 \%$ more selected $Z Z$ candidates than real $Z Z$ events.
Here a different shape can be seen and this could, for example, be used to optimize the selection. It was also observed that the $p_{T}(Z)$ spectrum becomes harder with an increasing value of $n$. A detailed study of the selection should, however, include proper detector simulation and event reconstruction. This will not be covered here, but would be a natural continuation of this work.

The same selection was used to investigate the case of spin-1 unparticle emission. Here the $Z+U$ signal was generated using the parameter points $d_{U}=1.4,1.6$ and 2.0 in accordance with [17] and $\lambda=1$ was kept fixed. Despite the fact that the unparticle mass spectrum shown in equation (3.2) is the same as in the spin-2 case, the spin- 1 matrix element implies a much softer $m_{U}$ as well as $p_{T}(Z)$ distribution. The $p_{T}(Z)$ distribution is illustrated in the lower plot of Fig. 5.2, which shows spin-1 unparticles with $d_{U}=2$ compared to the SM $Z Z$ distribution. For this reason the detection efficiency is significantly lower than in the spin2 case and, even with large amounts of data, the prospects seem poor for reaching significantly beyond the LEPII limits [17] using the $Z+U$ channel at the LHC. 

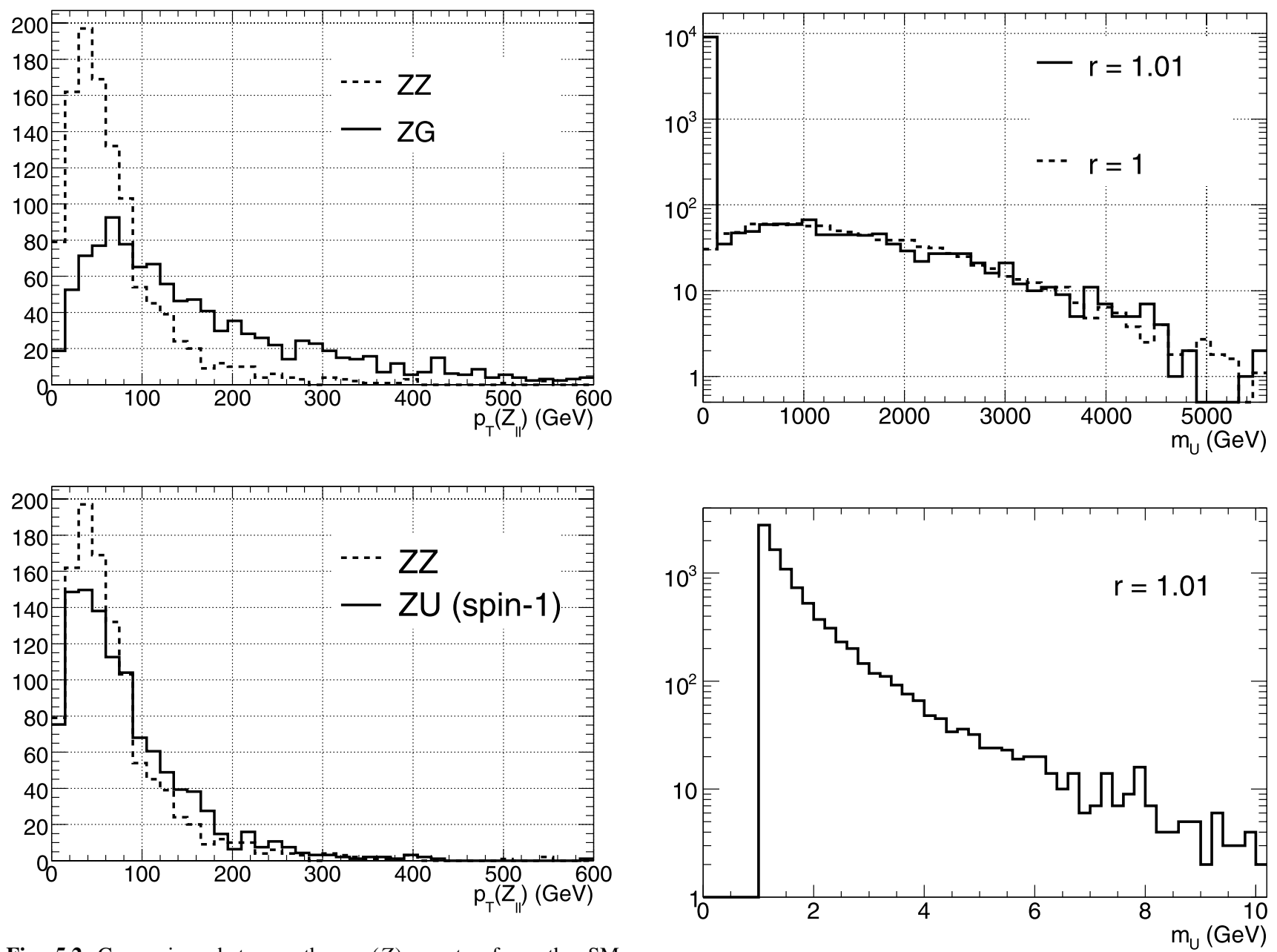

Fig. 5.2 Comparison between the $p_{T}(Z)$ spectra from the SM $Z Z \rightarrow \ell \ell \nu v$ and the $Z+G / U$ processes including truncation. The upper plot corresponds to a $Z+G$ signal with $n=2$ and $M_{D}=2 \mathrm{TeV}$. The lower plot corresponds to a spin- $1 Z+U$ signal with $d_{U}=2$ and $\Lambda_{U}=2 \mathrm{TeV}$. All distributions are based on events with two electrons or muons that pass the cuts 1 to 3 and have the same arbitrary normalization

The corresponding results for the spin-2 unparticle scenario with $\lambda=\lambda^{\prime}$ can be obtained simply by scaling the graviton results according to,

$\frac{\sigma_{U}}{\sigma_{G}}=\frac{A\left(d_{U}\right)}{S^{\prime}(n)} \cdot \lambda^{2}$

For this reason, the study of spin-2 unparticles was focused on deviating effects when $\lambda \neq \lambda^{\prime}$. Figure 5.3 shows the $m_{U}$ distributions of events generated with $d_{U}=2$ and $r\left[=\lambda^{\prime} / \lambda\right]=1.01$ as well as $r=1$. In the upper plot it can be seen that even for this small deviation from $\lambda=\lambda^{\prime}$, a large spike develops in the first bin. This spike is resolved in the lower plot and indicates an infra-red (IR) divergence of the cross section in the case of $\lambda \neq \lambda^{\prime}$. This is explained to some extent by the fact that the terms in the matrix element that cancel when $\lambda=\lambda^{\prime}$ are all proportional to powers of $1 / m_{U}^{2}$. For this reason also the total inclusive cross

Fig. 5.3 The (spin-2) unparticle mass spectra for $d_{U}=2$ with $r=1$ and $r=1.01$. The histograms are arbitrarily normalized. The distributions in the upper plot, however, have the correct ratio with respect to the total cross sections. The cut at $1 \mathrm{GeV}$ in the lower plot is a free parameter of the MC implementation.

section increases rapidly when the couplings are not equal. Unlike the spin- 1 case, the events in the soft $m_{U}$ tail have a significantly harder $p_{T}(Z)$ spectrum than the SM $Z Z$ process. This indicates that the $Z+U$ channel could also be a useful complement when investigating scenarios with $\lambda \neq \lambda^{\prime}$.

\section{Conclusions}

Models with extra dimensions have attracted much interest as candidates for potential physics beyond the SM. One of the most popular phenomena is real graviton emission and it has been pointed out earlier that graviton emission can be seen as a special case of the so-called unparticle emission process. This allows the same MC implementation to cover both scenarios and the parton level process 
$f \bar{f} \rightarrow Z / \gamma+G / U$ has been implemented in Pythia 8. In addition, $Z+G / U$ production at the LHC has been studied as a complement to the more conventional channels. This process was studied at generator level relative to the similar SM process $Z Z \rightarrow \ell \ell \nu v$. In order to get a handle on the experimental effects, a detailed analysis of the ATLAS $Z Z \rightarrow \ell \ell \nu v$ cross section measurement has been used as a reference. The study indicates that $Z+G$ production could be used for small values of $n$ to confirm a signal observed in the main channels. It will, however, require a relatively large amount of data. Spin-1 unparticle production at the LHC, on the other hand, seems more difficult to observe using the $Z+U$ channel. Spin- 2 unparticle events have the same characteristics as the graviton events if $\lambda=\lambda^{\prime}$. When these couplings are different an IR divergence is observed in the $m_{U}$ spectrum. This increases the total inclusive cross section and the $Z+U$ channel could provide a useful complement also in this scenario.

Acknowledgements The author wants to express special thanks to Kingman Cheung (Hsinchu) and Gian Giudice (CERN) for helpful discussions. Also Torbjörn Sjöstrand (Lund U.) is greatly acknowledged for his help with various Pythia related matters. This work was funded in the UK by STFC.

\section{Appendix A: Implementation in Pythia 8}

The processes $f \bar{f} \rightarrow Z+G / U$ and $f \bar{f} \rightarrow \gamma+G / U$ are implemented in two semi-internal classes called Sigma2ffbar2UZ and Sigma2ffbar2Ug. The classes include a number of standard Pythia methods which are called at different points during the event generation. For this reason they can be used by the main Pythia 8 library in the same way as for example the Sigma2bg2 Hb process in the main25.cc example of Pythia version 8.108.

The constructors takes 7 arguments in order to instantiate an object,

- int Spin, unparticle spin (1 or 2);

- bool Trunc, set true in order to truncate the contributing $\hat{s}$ values;

- bool Graviton, set true to use graviton specific settings according to (3.3), (3.4) and (3.5);

- double $d_{U}$, scale dimension parameter;

- double $\Lambda_{U}$, unparticle renormalization scale;

- double $\lambda$, unparticle coupling to SM;

- double Ratio, the ratio $\lambda^{\prime} / \lambda$ of the spin-2 matrix element [17].

If Graviton $=$ true, the $\lambda$ and Ratio values are overridden and set equal to one.

The graviton particle code 39 was used both in the case of graviton and unparticle emission. The graviton available in
Pythia has a Breit-Wigner distributed mass and the underlying Breit-Wigner shape can be adjusted in the main program by the following parameters,

pythia.readstring ("39:m0 = 50."); pythia.readString ("39:mWidth = 500.");

pythia.readstring ("39:mMin = 1."); pythia.readString ("39:mMax = 13910.");

The Breit-Wigner was re-weighted to a flat distribution by using the runBW3 weight in the sigmaHat ( ) method. By doing this the differential unparticle cross section in (3.2) ensures a proper mass distribution of the generated final events.

\section{Program Summary}

Code Location: sask.home.cern.ch/sask/gravunp100.tgz

Tested with: PYTHIA version 8.108

The .tar file contains:

README

main_gravunp.cc

Sigma2ffbar2UZ.h

Sigma2ffbar2Ug.h

Sigma2ffbar2UX.cc

\section{References}

1. N. Arkani-Hamed, S. Dimopoulos, G. Dvali, Phys. Lett. B 429, 263 (1998)

2. I. Antoniadis, N. Arkani-Hamed, S. Dimopoulos, G. Dvali, Phys. Lett. B 436, 257 (1998)

3. G.F. Giudice, R. Rattazzi, J.D. Wells, Nucl. Phys. B 544, 3 (1999)

4. E.A. Mirabelli, M. Perelstein, M.E. Peskin, Phys. Rev. Lett. 82, 2236 (1999)

5. S. Ask, in Proceedings of 32nd International Conference on High Energy Physics (ICHEP04), vol. 2 (World Scientific, Hackensack, 2005), p. 1289. arXiv:hep-ex/0410004

6. The ALEPH, DELPHI, L3 (OPAL Collaboration and the LEP Exotica Working Group), LEP Exotica WG 2004-03, ALEPH 2004007 PHYSICS 2004-006, DELPHI 2004-033 CONF 708, L3 Note 2798, OPAL Technical Note TN743 and references therein

7. R. Barate et al. (ALEPH Collaboration), Eur. Phys. J. C 28, 1 (2003)

8. J. Abdallah et al. (DELPHI Collaboration), Eur. Phys. J. C 38, 395 (2005)

9. P. Achard et al. (L3 Collaboration), Phys. Lett. B 587, 16 (2004)

10. G. Abbiendi et al. (OPAL Collaboration), Eur. Phys. J. C 18, 253 (2000)

11. V.M. Abazov et al. (D0 Collaboration). Phys. Rev. Lett. 101, 011601 (2008). http://www-d0.fnal.gov/Run2Physics/WWW/ results/np.htm

12. A. Abulencia et al. (CDF Collaboration), Phys. Rev. Lett. 97, 171802 (2006). http://www-cdf.fnal.gov/physics/W08CDFResults. html

13. L. Randall, R. Sundrum, Phys. Rev. Lett. 83, 3370 (1999)

14. G.F. Giudice, T. Plehn, A. Strumia, Nucl. Phys. B 706, 455 (2005)

15. J. Abdallah et al. (DELPHI Collaboration), Eur. Phys. J. C (2009, accepted). CERN-PH-EP/2008-013 
16. H. Georgi, Phys. Rev. Lett. 98, 221601 (2007)

17. K. Cheung, W.Y. Keung, T.C. Yuan, Phys. Rev. D 76, 055003 (2007)

18. T. Sjöstrand, S. Mrenna, P. Skands, J. High Energy Phys. 05, 026 (2006)

19. T. Sjöstrand, S. Mrenna, P. Skands, Comput. Phys. Commun. 178, 852 (2008)
20. K. Cheung, W.Y. Keung, Phys. Rev. D 60, 112003 (1999)

21. M.A. Stephanov, Phys. Rev. D 76, 035008 (2007)

22. G. Aad et al. (ATLAS Collaboration), CERN-OPEN-2008-020. arXiv:0901.0512 